Check for updates

Cite this: RSC Adv., 2018, 8, 8631

Received 23rd November 2017 Accepted 18th February 2018

DOI: $10.1039 / c 7 r a 12697 b$

rsc.li/rsc-advances

\section{A polymer inclusion membrane composed of the binary carrier PC-88A and Versatic 10 for the selective separation and recovery of Sc}

\author{
Maha Sharaf, ${ }^{a}$ Wataru Yoshida, (D) ${ }^{a}$ Fukiko Kubota, ${ }^{a}$ Spas D. Kolev (D) cd \\ and Masahiro Goto (D) *ab
}

\begin{abstract}
This study reports on the selective separation of scandium (Sc) from other rare earth metals (REMs) using a polymer inclusion membrane (PIM). The PIM prepared with PC-88A (2-ethylhexyl hydrogen-2ethylhexylphosphonate) alone as the carrier showed high extractability but the poor back-extraction of the extracted $\mathrm{Sc}^{3+}$ ions did not allow the transport of these ions to the receiving solution of a membrane transport system. To overcome this problem, a novel approach was introduced using a mixture of carriers that allowed $\mathrm{Sc}^{3+}$ transport into the receiving solution. A cellulose triacetate (CTA) based PIM containing both PC-88A and Versatic 10 (decanoic acid) as carriers and dioctyl phthalate (DOP) as a plasticizer was prepared for the selective separation of $\mathrm{Sc}^{3+}$ from other REM ions in nitrate media. The membrane composition was optimized and the effect of operational parameters such as $\mathrm{pH}$ of the feed solution and composition of the receiving solution was explored. The flux at the membrane/feed solution interface was found to depend significantly on the carrier concentration in the PIM, pH of the feed solution and the receiving solution acidity. The newly developed PIM allowed quantitative and selective transport of $\mathrm{Sc}^{3+}$ thus demonstrating its suitability for the selective recovery of this metal.
\end{abstract}

\section{Introduction}

The demand for rare earth metals (REMs) has been steadily increasing due to their unique physical and chemical characteristics and extensive usage in various life sectors, e.g., adding a small amount of REMs to steel can improve its heat, corrosion and oxidation resistance. ${ }^{1}$ Scandium is a REM, which has an essential role in many application areas such as aluminium alloys, semiconductors and fuel-cells. ${ }^{2}$ The price of scandium is considerable and it is produced mainly as a by-product, therefore, there is an urgent need for the development of dedicated and efficient separation techniques to sustain the growing demand for this metal. Solvent extraction is one of the industrially established separation methods to recover REMs from aqueous solutions. ${ }^{3,4}$ However, it still has some significant drawbacks such as requiring relatively complex equipment, consuming high amounts of solvents, involving multi-stage

\footnotetext{
${ }^{a}$ Department of Applied Chemistry, Graduate School of Engineering, Kyushu University, 744 Motooka, Fukuoka 819-0395, Japan. E-mail: m-goto@mail.cstm. kyushu-u.ac.jp; Fax: +81 92802 2810; Tel: +8192802 2806

${ }^{b}$ Center for Future Chemistry, Kyushu University, 744 Motooka, Fukuoka 819-0395, Japan

'School of Chemistry, The University of Melbourne, Victoria 3010, Australia

${ }^{d}$ Centre for Aquatic Pollution Identification and Management (CAPIM), The University of Melbourne, Victoria 3010, Australia
}

extraction processes, extensive energy consumption, and limited ligand selection. ${ }^{5}$

In recent years, liquid membrane technologies have attracted much attention in the separation and recovery of metal ions from aqueous solutions ${ }^{6}$ because of their high energy efficiency, flexibility, low cost, easily satisfying environmental pollution regulations, and offering controlled membrane permeability. There are several kinds of liquid membranes for metal separation from aqueous liquors, such as supported liquid membranes (SLMs), emulsion liquid membranes (ELMs), bulk liquid membranes (BLMs), and polymer inclusion membranes (PIMs). ${ }^{7}$ In the present study, we have focused on PIMs due to several important advantages they offer compared to the other types of membrane mentioned above.

PIMs are liquid membranes which consist of a base polymer (usually poly(vinyl chloride), PVC or cellulose triacetate, CTA), a carrier (often a commercial solvent extractant) which is immobilized between the entangled chains of the base polymer, and a plasticizer, if necessary. ${ }^{8-10}$ PIM-based separation is considered as a highly promising technique because of the high stability, selectivity, efficiency, and durability of PIMs. ${ }^{11}$ The outstanding performance of PIMs in comparison with the other types of liquid membranes is expected to lead to the development of novel industrial separation processes in the near future. PIMs have already been shown to be applicable to the separation of some REMs, ${ }^{12-14}$ actinides ${ }^{15,16}$ as well as other metals. ${ }^{17-24}$ However, there have been no reports so far on scandium 
separation using PIMs because of the lack of suitable extractants/carriers. There are several good extractants with

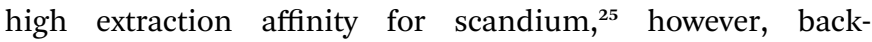
extraction is known to be difficult when such extractants are used. To establish a successful membrane separation system, the efficiency of back-extraction is equally important as that of extraction.

In our previous solvent extraction study, ${ }^{\mathbf{2 6}}$ we found that a binary extractant composed of PC-88A and Versatic 10 showed a good performance for both the selective extraction and efficient back-extraction of scandium. The present paper reports on the development, optimization and characterization of the first PIM for the selective separation of the scandium ion from other REM ions.

\section{Experimental}

\subsection{Reagents and chemicals}

PC-88A and Versatic 10 were supplied by Daihachi Chemical (Osaka, Japan) and Japan Epoxy Resin (Tokyo, Japan) (currently Mitsubishi Chemical Corporation), and used without further purification. CTA used as the PIM basepolymer, dioctylphthalate (DOP) and 2-nitrophenyloctyl ether (2NPOE) used as plasticizers were purchased from Sigma-Aldrich (St Louis, USA). The salts of the REMs studied were provided by Aldrich and used in the preparation of the feed solutions. Sulfuric acid, nitric acid and ammonium nitrate were purchased from Kishida Chemical (Osaka, Japan).

\subsection{Membrane preparation}

In this study, PIMs using CTA as the base-polymer, and dioctylphthalate (DOP) and 2-nitrophenyloctyl ether (2NOPE), used as plasticizers were prepared as previously described. ${ }^{27} \mathrm{~A}$ homogeneous solution containing the required amounts of CTA (20-70 wt $\%)$, the plasticizer (0-40 wt $\%$ ) and the mixed carrier (0-40 wt\%) with a total mass of $400 \mathrm{mg}$ was prepared in $10 \mathrm{~mL}$ dichloromethane. The ratio of PC-88A and Versatic 10 was also varied. The solution was then poured into a $7.5 \mathrm{~cm}$ in diameter glass ring positioned on a flat glass plate, and covered with a filter paper and a watch glass to allow the slow evaporation of the solvent overnight. The prepared membranes were transparent with a soft surface and a good mechanical strength as shown in Fig. 1. Membrane thickness was measured using Digimatic Micrometer MDC-25 MX (Mitutoyo, Japan) and the corresponding value was calculated as the average of 10 spots along the membrane diameter. The average thickness of the membranes used in all experiments was $60 \pm 5 \mu \mathrm{m}$.

\subsection{Membrane extraction and back-extraction experiments}

The PIM extraction experiments were conducted by immersing a circular segment of the membrane with an average mass of $80 \mathrm{mg}$ in a $50 \mathrm{~mL}$ of an aqueous feed solution in a glass flask covered with Parafilm. To evaluate the effect of parameters on the PIM performance, all experiments were performed at

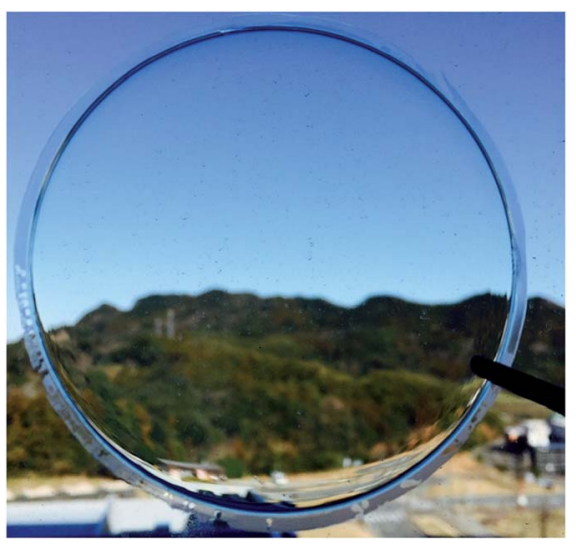

Fig. 1 A photo image of a prepared membrane.

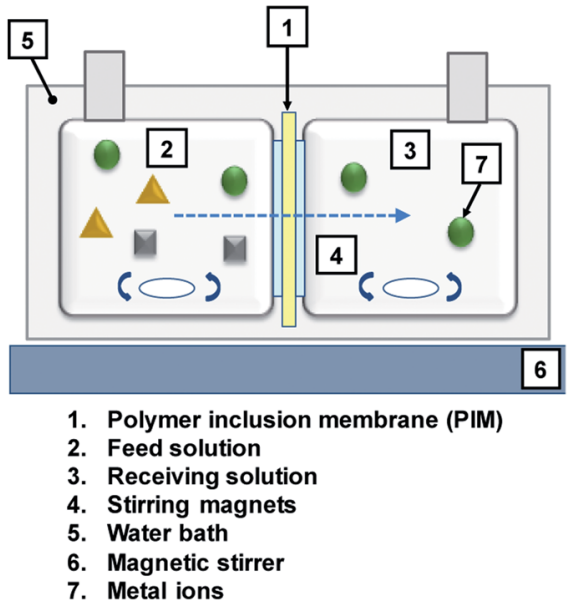

Fig. 2 Schematic diagram of the PIM transport cell.

a constant $\mathrm{pH}$ of 4 , unless otherwise stated. All extraction experiments were performed at a stirring rate of $90 \mathrm{rpm}$ at $25{ }^{\circ} \mathrm{C}$ using a thermostated orbital shaker (EYELA NTS-4000, Tokyo Rikakikai Co., Ltd., Tokyo, Japan). The aqueous feed solutions were prepared by dissolving metal salts in mixtures of $0.1 \mathrm{M} \mathrm{HNO}_{3}$ and $0.1 \mathrm{M} \mathrm{NH}_{4} \mathrm{NO}_{3}$ solutions. The $\mathrm{pH}$ of the feed solutions was adjusted by varying the ratio of the $\mathrm{HNO}_{3}$ and $\mathrm{NH}_{4} \mathrm{NO}_{3}$ solutions. The $\mathrm{pH}$ during the extraction experiments was measured at predetermined times by using a $\mathrm{pH}$ meter (H-M-60 G, DKK-TOA, Japan). Samples (1 mL) were taken periodically from the feed solution and replaced with the same volume of the initial feed solution. Metal concentrations were analyzed by inductively coupled plasma-atomic emission spectrometry (ICP-AES, Optima 8300; Perkin Elmer, USA).

The kinetics of the PIM extraction was described by the following first order equation:

$$
\ln \left(\frac{\left[\mathrm{M}^{3+}\right]_{\mathrm{f}}}{\left[\mathrm{M}^{3+}\right]_{\mathrm{f}, 0}}\right)=-k t
$$

where $t$ is the time $(\mathrm{h}),\left[\mathrm{M}^{3+}\right]_{\mathrm{f}, 0}$ and $\left[\mathrm{M}^{3+}\right]_{\mathrm{f}}$ are the concentrations of the REM ion in the feed solution at times 0 (original solution) 
and $t$, respectively, and $k$ is the kinetic rate constant $\left(\mathrm{h}^{-1}\right)$ for the metal uptake into the membrane.

The back-extraction experiments were conducted by immersing the loaded with a REM ion membrane into a sulfuric acid receiving solution.

\subsection{Membrane transport experiment}

Metal transport experiments were performed using a transport system consisting of two jacketed glass compartments with the PIM sandwiched between them as shown in Fig. 2. The membrane apparatus is designed for analyzing a transport behavior, and the same apparatus was used in the study of supported liquid membranes (SLMs) experiments. ${ }^{28,29}$ The two compartments were filled with the feed and receiving solutions (50 $\mathrm{mL}$ each). The diameter of the membrane exposed to the solutions was $25.0 \mathrm{~mm}$ (effective surface area of $4.9 \times 10^{-4} \mathrm{~m}^{2}$ ). The solutions in both compartments were mechanically stirred at $300 \mathrm{rpm}$ during the experiments using magnetic stirrers $(\mathrm{KH}-$ 55D, Vidrex, Japan) and small crosshead magnetic stirring bars $(8 \times \phi 10)$. Samples $(1 \mathrm{~mL})$ were taken from the ports of both glass compartments at predetermined times and analyzed by ICP-AES. The samples were replaced with the same volume of the corresponding fresh solutions. Temperature of both compartments was kept constant at $25{ }^{\circ} \mathrm{C}$ by continuously circulating water through the compartments' jackets from a water bath with a thermoregulator (RCB-1200, EYELA, Japan). The feed solution containing initially $0.1 \mathrm{mM}$ metal ion(s) and the receiving solution were prepared as described earlier. As in the extraction experiments, it was assumed that the transport kinetics was first order (eqn (1)). The permeability coefficient $(P)$, initial flux $J_{0}\left(\mathrm{~mol} \mathrm{~m}^{-2} \mathrm{~s}^{-1}\right)$ and recovery factor $\mathrm{RF}(\%)$ were determined by eqn (2)-(4), respectively.

$$
\begin{gathered}
P=\frac{V}{A} k \\
J_{0}=P\left[\mathrm{M}^{3+}\right]_{0}^{\mathrm{f}} \\
\operatorname{RF}(\%)=\frac{\left[\mathrm{M}^{3+}\right]_{0}^{\mathrm{f}}-\left[\mathrm{M}^{3+}\right]^{\mathrm{f}}}{\left[\mathrm{M}^{3+}\right]_{0}^{\mathrm{f}}} 100
\end{gathered}
$$

where $V\left(\mathrm{~m}^{3}\right)$ is the volume of feed solution, $A\left(\mathrm{~m}^{2}\right)$ is the membrane surface area exposed to each of the two solutions, and superscript $f$ refers to the feed solution.

\subsection{Membrane characterization}

The morphology of the PIMs studied was examined by imaging their cross-sections using scanning electron microscopy (SEM, Inspect S50 XT microscope, type: FB 2017/12-FEI, USA). The membrane samples were first immersed into liquid nitrogen for $15 \mathrm{~min}$, then were cut into small rectangular pieces to be fixed onto the sample holder, and finally were coated with palladium (MSP-15 Magnetron Sputter Hitachi, Japan). SEM images at two different scales were taken.

\section{Results and discussion}

\subsection{Optimization of the PIM composition}

The optimization of the composition of the CTA-based PIM containing PC-88A and Versatic 10 was based on extraction and back-extraction experiments involving PIMs with different compositions.

3.1.1 Carrier. Initially, PIMs containing either PC-88A or Versatic 10 were studied. Fig. 3(a) shows the transient concentrations of REM ions $\left(\left[\mathrm{M}^{3+}\right]\right)$ of interest in the feed solution normalized with respect to their initial concentrations $\left(\left[\mathrm{M}^{3+}\right]_{0}\right)$ during extraction experiments at $\mathrm{pH} 1$ involving PIMs containing different concentrations of the extractant PC-88A and 30\% of the plasticizer DOP. The results showed that $\mathrm{Sc}^{3+}$ was selectively extracted into the membranes and the extraction rate and amount extracted increased with increasing the PC-88A concentration. Upon increasing PC-88A concentration in the PIM up to $10 \%$, the extracted amount of $\mathrm{Sc}^{3+}$ gradually increased and quantitative extraction of $\mathrm{Sc}^{3+}$ into a PIM containing $10 \%$ PC-88A was achieved after $24 \mathrm{~h}$ of extraction. In experiments with feed solutions at $\mathrm{pH} 4$ other REMs were also extracted and this lowered the extraction rate and the amount of $\mathrm{Sc}^{3+}$ extracted. However, it was difficult to back-extract $\mathrm{Sc}^{3+}$ from the PC-88A containing PIMs regardless of the concentration of PC-88A as shown in Fig. 3(b) where the transient concentration
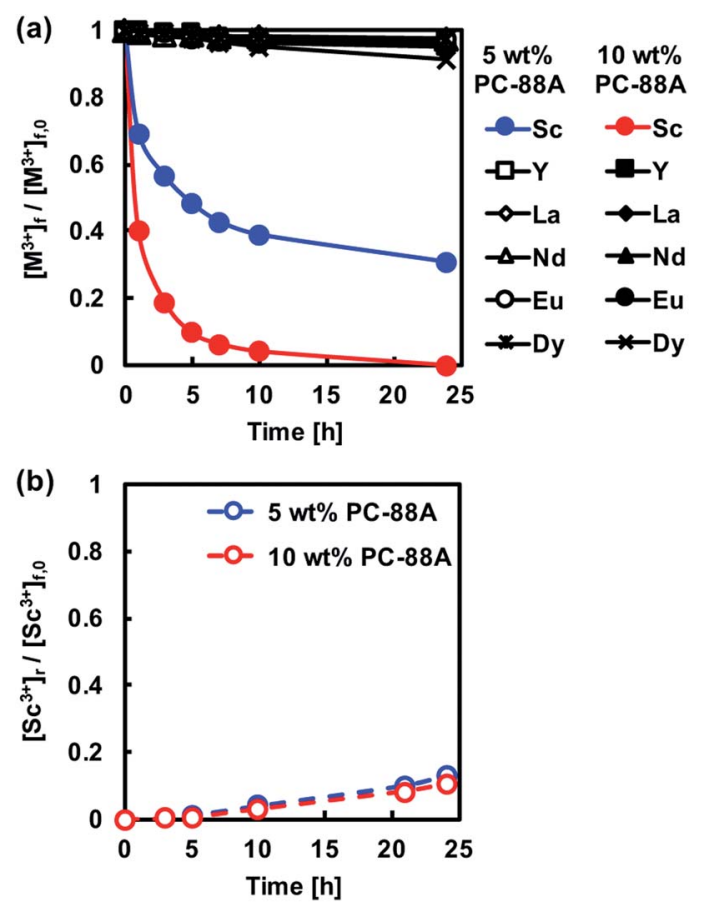

Fig. 3 (a) Normalized transient concentrations in the feed solution of the REM ions studied during the PIM extraction experiments. (b) Normalized transient $\mathrm{Sc}^{3+}$ concentration in the $1 \mathrm{M}$ sulfuric acid receiving solution during the PIM back-extraction experiments. Experimental conditions: CTA-based PIMs composition - 5 or $10 \mathrm{wt} \%$ PC-88A and $30 \mathrm{wt} \%$ DOP; PIM mass and thickness $-80 \mathrm{mg}$ and $60 \pm 5$ $\mu \mathrm{m}$, respectively; feed solution volume, initial composition and $\mathrm{pH}-$ $50 \mathrm{~mL}, 0.1 \mathrm{mM}$ of each of the REM ions studied and 1, respectively. 

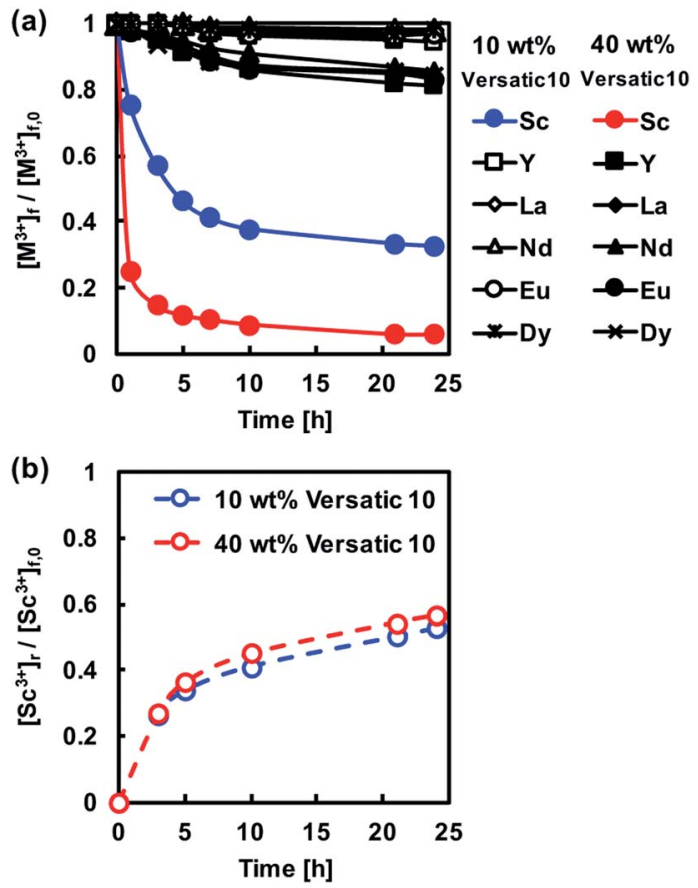

Fig. 4 (a) Normalized transient concentrations in the feed solution of the REM ions studied during the PIM extraction experiments. (b) Normalized transient $\mathrm{Sc}^{3+}$ concentration in the $1 \mathrm{M}$ sulfuric acid receiving solution during the PIM back-extraction experiments. Experimental conditions: CTA-based PIMs composition - 10 or $40 \mathrm{wt} \%$ Versatic 10 and $30 \mathrm{wt} \%$ DOP; PIM mass and thickness $-80 \mathrm{mg}$ and $60 \pm 5 \mu \mathrm{m}$, respectively; feed solution volume, initial composition and $\mathrm{pH}-50 \mathrm{~mL}, 0.1 \mathrm{mM}$ of each of the REM ions studied, 4 respectively

of $\mathrm{Sc}^{3+}$ is normalized with respect to the initial concentration in the feed solution $\left(\left[\mathrm{Sc}^{3+}\right]_{0}\right)$. The inability to back-extract quantitatively $\mathrm{Sc}^{3+}$ from the PIM would be a serious obstacle in developing a PIM-based separation technology for this metal.

The membrane extraction of REM ions was also carried out using PIMs with Versatic 10 alone as the carrier. The extraction ability of the extractant Versatic 10 for $\mathrm{Sc}^{3+}$ is considerably lower than that of $\mathrm{PC}-88 \mathrm{~A}^{25}$ and therefore the extraction experiments involving Versatic 10-based PIMs were conducted at feed solution $\mathrm{pH}$ of 4 rather than 1 (Fig. 4(a)). As expected, the backextraction of $\mathrm{Sc}^{3+}$ from the Versatic 10-based PIMs was found to be better than that from the PC-88A-based membranes (Fig. 4(b)).

It has been proven that the use of binary extractants in solvent extraction systems ${ }^{30}$ can improve their ability to selectively and reversibly extract specific target metal ions. In our previous study, we have found that the binary extractants PC$88 \mathrm{~A}$ and Versatic 10 are suitable for both the selective extraction and efficient back-extraction of $\mathrm{Sc}^{3+} .{ }^{26}$ It was confirmed that $\mathrm{Sc}^{3+}$ is extracted via the exchange by three protons in the binary extractant system. The reaction seemed to involve predominantly PC-88A, and Versatic 10 was considered to reduce the affinity of $\mathrm{PC}-88 \mathrm{~A}$ to $\mathrm{Sc}^{3+}$. This result improved the backextraction efficiency. Therefore, the possibility of using this binary extractant as the carrier of a CTA-based PIM was examined as a part of the present study. It was expected that the
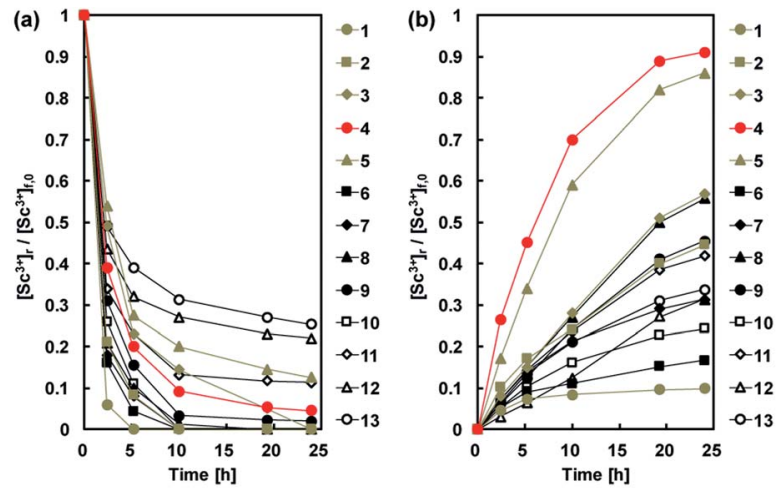

\begin{tabular}{c|ccccc|cccc|cc|cc}
\multicolumn{11}{c}{ Binary carrier composition (wt\%) } \\
\hline No. & 1 & 2 & 3 & 4 & 5 & 6 & 7 & 8 & 9 & 10 & 11 & 12 & 13 \\
\hline PC-88A & 20 & 10 & 6 & 4 & 2 & 15 & 10 & 6 & 4 & 10 & 4 & 5 & 2 \\
Versatic 10 & 20 & 30 & 34 & 36 & 38 & 15 & 20 & 24 & 26 & 10 & 16 & 5 & 8 \\
\hline Total & \multicolumn{10}{c|}{} & 40 & \multicolumn{10}{c|}{30} & 20 & \multicolumn{10}{c}{10} \\
\hline
\end{tabular}

Fig. 5 (a) Normalized transient $\mathrm{Sc}^{3+}$ concentration in the feed solution during the PIM extraction experiments. (b) Normalized transient $\mathrm{Sc}^{3+}$ concentration in the $1 \mathrm{M}$ sulfuric acid receiving solution during the PIM back-extraction experiments. Experimental conditions: CTA-based PIMs composition - binary extractant (composition in the above table) and 30 wt\% DOP. Remaining experimental conditions as in Fig. 4.

presence of Versatic 10 would improve the stripping efficiency of $\mathrm{Sc}^{3+}$ from the PIM with the binary extractant compared to the PIM containing only PC-88A while keeping the high selectivity of the latter membrane.

The extraction and back-extraction characteristics of CTAbased PIMs containing $30 \mathrm{wt} \%$ DOP as plasticizer and both PC-88A and Versatic 10 as the binary carrier in concentrations equal to $10 \mathrm{wt} \%, 20 \mathrm{wt} \%, 30 \mathrm{wt} \%$, and $40 \mathrm{wt} \%$ were studied. The ratio between the two extractants was varied, while all the extraction experiments were conducted with the same initial feed solution concentration of $0.1 \mathrm{mM}$ and $\mathrm{pH}$ of 4 . Best extraction was achieved at this $\mathrm{pH}$ value in a series of experiments where the initial $\mathrm{pH}$ was varied between 1 and 4 . It should be noted that in the course of the extraction process the $\mathrm{pH}$ decreased from 4 to around 3.3. As shown in Fig. 5, the extraction of $\mathrm{Sc}^{3+}$ was enhanced by increasing the PC-88A concentration of the membrane, however, the back-extraction of $\mathrm{Sc}^{3+}$ was depressed as a result of this. Moreover, increasing the Versatic 10 percentage over that of PC-88A was found to be effective for improving the back-extraction efficiency. Both quantitative extraction and back-extraction were achieved with a PIM containing $40 \mathrm{wt} \%$ carrier $(4 \mathrm{wt} \%$ PC- $88 \mathrm{~A}+36 \mathrm{wt} \%$ Versatic 10$), 30 \mathrm{wt} \%$ CTA and $30 \mathrm{wt} \%$ DOP. PIM with concentration

Table 1 Rate constant $(k)$ values for $\mathrm{Sc}^{3+}$ extraction in PIMs of different carrier composition. Remaining experimental conditions as in Fig. 4

\begin{tabular}{lll}
\hline Carrier compositions & DOP $(\mathrm{wt} \%)$ & $k\left[\mathrm{~h}^{-1}\right]$ \\
\hline $10 \mathrm{wt} \%$ PC-88A* & 30 & 0.29 \\
$40 \%$ Versatic 10 & 30 & 0.36 \\
$4 \mathrm{wt} \%$ PC-88A + 36 wt\% Versatic 10 & 30 & 0.55 \\
$4 \mathrm{wt} \%$ PC-88A + 36 wt\% Versatic 10 & 40 & 0.86
\end{tabular}



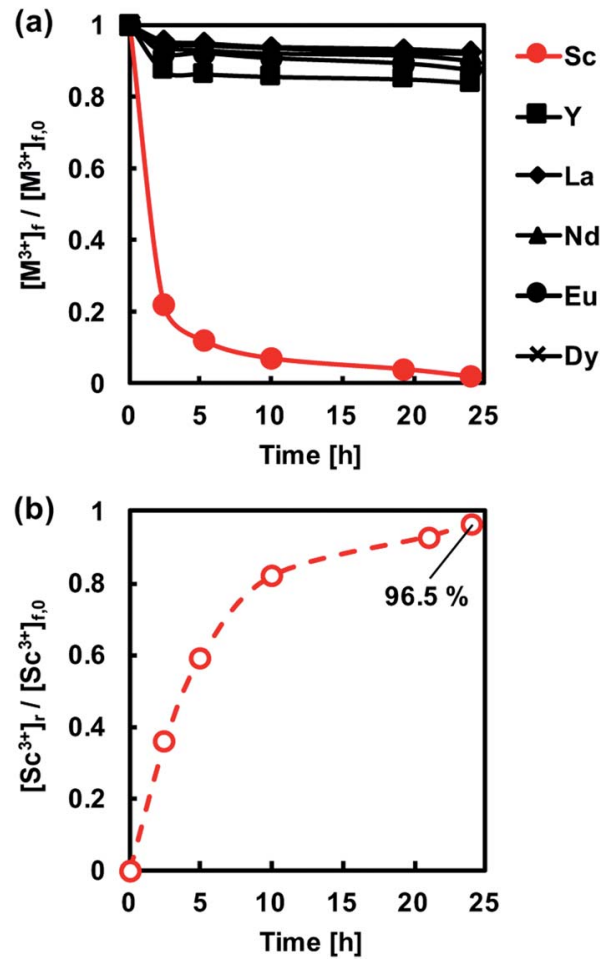

Fig. 6 (a) Normalized transient concentrations in the feed solution of the REM ions studied during the PIM (4 wt\% PC-88A and $36 \mathrm{wt} \%$ Versatic 10) extraction experiments. (b) Normalized transient $\mathrm{Sc}^{3+}$ concentration in the $1 \mathrm{M}$ sulfuric acid receiving solution during the PIM back-extraction experiments. Remaining experimental conditions as in Fig. 4 except for the DOP concentration which was $40 \mathrm{wt} \%$.

of the carrier greater than $40 \mathrm{wt} \%$, was found to be mechanically unstable and therefore the combination of $4 \mathrm{wt} \% \mathrm{PC}-88 \mathrm{~A}$ and $36 \mathrm{wt} \%$ Versatic 10 was selected as the optimum carrier composition. The results mentioned above demonstrated the importance of controlling the ratio between the two extractants for achieving efficient extraction and back-extraction of $\mathrm{Sc}^{3+}$. The initial rate constant $(k)$ values calculated by eqn (1) for different carrier compositions in the membrane are summarized in Table 1.

3.1.2 Plasticizer. CTA-based PIMs containing 4 wt $\%$ PC-88A and 36 wt $\%$ Versatic 10 as the binary extractant and DOP or 2NPOE as the plasticizer in the concentration range of $0-40 \mathrm{wt} \%$ were studied. The experimental results suggested that the membrane extraction rate increased upon increasing the percentage of the plasticizer in the membrane (data not shown). The best extraction and back-extraction efficiency was achieved when using $40 \mathrm{wt} \%$ DOP. The extraction and back-extraction results obtained at this PIM composition are shown in Fig. 6, and the initial extraction rate constant values are listed in Table 1. It can be expected that by increasing the plasticizer concentration, the viscosity of the membrane liquid solution will decrease thus providing more opened polymer structure and lower membrane diffusion coefficients. ${ }^{31}$ The PIMs prepared with 2 NPOE as the plasticizer were less flexible than those containing DOP. In addition, DOP is the less expensive plasticizer. Therefore, DOP was considered to be the more suitable plasticizer for preparing the PIMs with optimal composition.
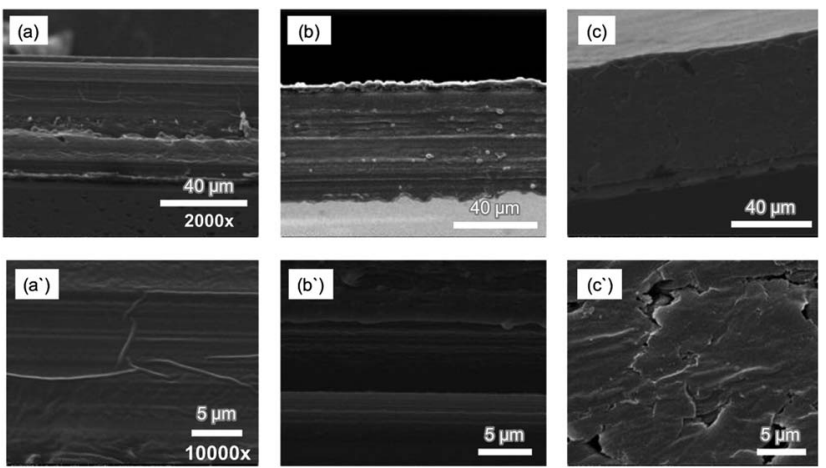

Fig. 7 SEM cross section micrographs at two scales: magnification $2000 \times$ and $10000 \times$ of CTA-based PIMs with 30 wt\% DOP (a and $a^{\prime}$ ); 4 wt\% PC-88A, 36 wt\% Versatic 10 and 30 wt\% DOP (b and $b^{\prime}$ ); and 4 wt\% PC-88A and 36 wt\% Versatic 10 (c and $c^{\prime}$ ).

3.1.3 Stripping reagent. Three different mineral acids $(\mathrm{HCl}$, $\mathrm{HNO}_{3}$ and $\mathrm{H}_{2} \mathrm{SO}_{4}$ ) were studied for their suitability as stripping reagents for $\mathrm{Sc}^{3+}$ and it was established that their stripping efficiency increased in the order $\mathrm{HNO}_{3}>\mathrm{HCl}>\mathrm{H}_{2} \mathrm{SO}_{4}$. However, when we used the nitric and hydrochloric acids as the receiving solution in membrane transport experiments, the $\mathrm{pH}$ values in the feed solution drastically dropped down. Therefore, in the membrane transport experiments, sulfuric acid was selected due to its high hydrophilicity, which protected the acid transport from the receiving solution to the feed one.

\subsection{Membrane characterization by SEM}

The morphology of PIMs with different compositions was investigated using high resolution SEM. Fig. 7 shows SEM crosssectional images at two scales; magnification $2000 \times$ and $10000 \times$ of CTA-based PIMs containing DOP (a and $\mathrm{a}^{\prime}$ ); PC-88A, Versatic 10 and DOP (b and $b^{\prime}$ ); and PC-88A and Versatic 10 without DOP $\left(c\right.$ and $\left.c^{\prime}\right)$. All the membranes shown in Fig. 7 were transparent as shown in Fig. 1. The SEM images of CTA-based PIM with only DOP revealed a homogenous and uniform membrane structure (Fig. $7\left(a^{\prime}\right)$ ). The SEM image of the PIM binary system composed of $4 \mathrm{wt} \% \mathrm{PC}-88 \mathrm{~A}$ and $36 \mathrm{wt} \%$ Versatic 10 with $30 \mathrm{wt} \%$ DOP as the plasticizer, which offered the best $\mathrm{Sc}^{3+}$ ion transport, exhibited a well-oriented multilayer fibrous structure (Fig. $7\left(b^{\prime}\right)$ ). The PIM with the same binary carrier but without DOP (Fig. $7\left(c^{\prime}\right)$ ) is characterized by a non-uniform cracked structure thus illustrating the critical role of the plasticizer in forming a uniform and homogeneous PIM and explaining the poor permeability of this PIM. Consequently, it can be concluded that by adding a plasticizer, both the uniformity and permeability of the membranes increases significantly. Moreover, the PIMs prepared with DOP were thinner and with a low density (compared (b) to (c)) that would further promote high membrane permeability. These results are in agreement with the study of Manzak et al. ${ }^{32}$ about the role of the plasticizer in constructing more flexible and softer PIMs and in minimizing the intermolecular interaction forces in their polymer scaffolds thus resulting in higher membrane permeability. 
Table 2 Effect of acid type and concentration in the receiving solution on the transport efficiency for the $\mathrm{Sc}^{3+}$ ion and the $\mathrm{pH}$ change of the feed solution after $24 \mathrm{~h}$ of transport. Remaining experimental conditions as in Fig. 6

\begin{tabular}{|c|c|c|c|c|}
\hline \multirow[b]{2}{*}{ Acids } & \multicolumn{2}{|l|}{$\mathrm{pH}^{\mathrm{f}}$} & \multirow{2}{*}{$\begin{array}{l}\text { Remaining } \% \text { of } \mathrm{Sc}^{3+} \\
\text { in the feed } \\
\text { solution }\end{array}$} & \multirow{2}{*}{$\begin{array}{l}\text { Recovery\% of } \mathrm{Sc}^{3+} \\
\text { in the receiving } \\
\text { solution }\end{array}$} \\
\hline & $t=0 \mathrm{~h}$ & $t=24 \mathrm{~h}$ & & \\
\hline $1 \mathrm{M} \mathrm{H}_{2} \mathrm{SO}_{4}$ & 4 & 3.33 & 15.7 & 35.7 \\
\hline $2 \mathrm{M} \mathrm{H}_{2} \mathrm{SO}_{4}$ & 4 & 3.21 & 2.5 & 39.8 \\
\hline $3 \mathrm{M} \mathrm{H}_{2} \mathrm{SO}_{4}$ & 4 & 3.12 & 23.5 & 18.2 \\
\hline $1 \mathrm{M} \mathrm{HCl}$ & 4 & $<$ Zero & 59.7 & 12.5 \\
\hline $1 \mathrm{M} \mathrm{HNO}_{3}$ & 4 & $<$ Zero & 68.8 & 4 \\
\hline
\end{tabular}

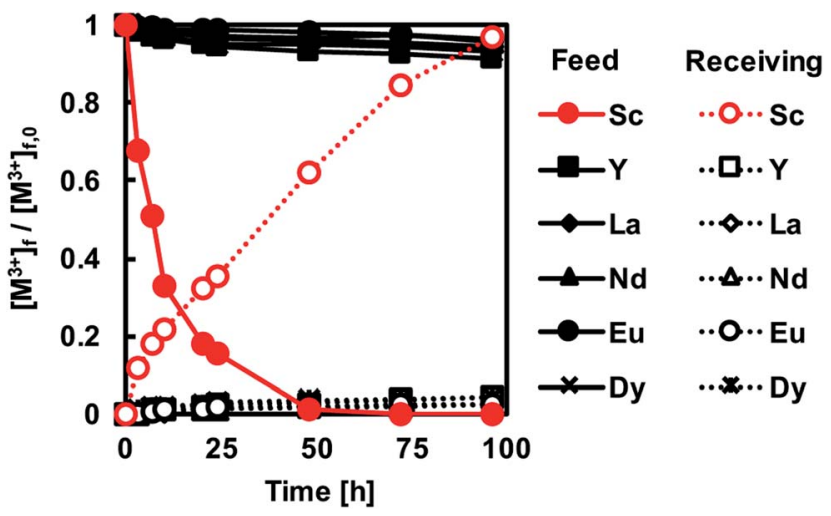

Fig. 8 Transport of REM ions across a PIM of optimal composition. Experimental conditions as in Fig. 6.

\subsection{Membrane transport experiments}

Membrane transport experiments of metal ions were conducted using PIMs with total binary carrier concentration of $40 \mathrm{wt} \%$, where the ratio between PC-88A and Versatic 10 was varied. When maintaining the percentage of Versatic 10 constant and varying the PC-88A percentage between 1 and $3 \mathrm{wt} \%$, incomplete extraction of $\mathrm{Sc}^{3+}$ was observed from a nitrate acid feed solution at pH 4, while more than $6 \mathrm{wt} \%$ PC-88A enhanced the transport of the other REM ions studied over that of $\mathrm{Sc}^{3+}$ into the $1 \mathrm{M}$ sulfuric acid receiving solution. Therefore, the membrane composition of $40 \mathrm{wt} \%$ binary extractant (4 wt\% PC$88 \mathrm{~A}+36 \mathrm{wt} \%$ Versatic 10$), 40 \mathrm{wt} \%$ DOP and $20 \mathrm{wt} \%$ CTA, already selected as optimal for the extraction and stripping of $\mathrm{Sc}^{3+}$, was found to be optimal for its transport as well.

A PIM with optimal composition mentioned above but without the plasticizer DOP showed very poor permeability for $\mathrm{Sc}^{3+}$ in transport experiments.

As described before, the extraction of $\mathrm{Sc}^{3+}$ proceeds via the exchange of three protons in the binary extractant system. ${ }^{26}$ Membrane transport of the metals is thought to proceed by the following mechanism: the extraction reaction with a cation exchange occurs at the membrane surface of the feed side, followed by the diffusion of the metal species in the membrane from the feed to the receiving side where the metal ion is recovered into the receiving solution.

The driving force in the membrane transport is the concentration gradient of the hydrogen ions between the feed and the receiving solution. As described above, the highly acidic conditions in the receiving solution were crucial for obtaining effective transport across the membrane. The percentage of $\mathrm{Sc}^{3+}$ recovery after a $24 \mathrm{~h}$ transport was about $36 \%$ when a $1 \mathrm{M} \mathrm{H}_{2} \mathrm{SO}_{4}$ receiving solution was used and this value slightly increased with increasing the $\mathrm{H}_{2} \mathrm{SO}_{4}$ concentration to $2 \mathrm{~mol} \mathrm{~L}^{-1}$. However, a decline of the transport efficiency was observed when $3 \mathrm{M}$ $\mathrm{H}_{2} \mathrm{SO}_{4}$ was used as shown in Table 2 . This result was contrary to the Le Chatelier's principle according to which with increasing of the concentration gradient of the hydrogen ions the extraction rate should increase accordingly. ${ }^{33}$ However, when the sulfuric acid concentration was higher than $2 \mathrm{~mol} \mathrm{~L}^{-1}$, coextraction of the acid was observed, which caused a decrease in the $\mathrm{pH}$ of the feed solution. When the receiving solution contained $1 \mathrm{M} \mathrm{HCl}$ or $1 \mathrm{M} \mathrm{HNO}_{3}$, very small amount of $\mathrm{Sc}^{3+}$ was recovered after a $24 \mathrm{~h}$ operation, as summarized in Table 2. The drop in the $\mathrm{pH}$ value in the feed solution to around zero after $6 \mathrm{~h}$ of transport was observed, which was due to the fast transport of acids into the feed solution.

Fig. 8 shows the transport behaviour of REM ions including $\mathrm{Sc}^{3+}$ through the CTA-based PIM with the optimal composition and a receiving solution containing $1 \mathrm{M} \mathrm{H}_{2} \mathrm{SO}_{4}$. It was found that $\mathrm{Sc}^{3+}$ concentration in the feed solution decreased relatively fast, while in the receiving solution, the concentration gradually increased. The difference in the rates of depletion and accumulation of $\mathrm{Sc}^{3+}$ in both solutions was considered to be the fact that the metal transport was dominated by the diffusion in the membrane. Therefore, the rate of accumulation of the metal ion in the receiving solution was slower in the initial stages of the transport process (Fig. 8). After $96 \mathrm{~h}$ from the start of the transport experiment, $\mathrm{Sc}^{3+}$ was quantitatively recovered in the receiving solution with a recovery factor of $96.7 \%$, and only a small amount of other REM ions was transported through the membrane. These results demonstrate that the binary carrier membrane containing PC-88A and Versatic 10 is suitable for the selective and quantitative recovery of $\mathrm{Sc}^{3+}$ from its nitrate solutions containing other REM ions into a $1 \mathrm{M} \mathrm{H}_{2} \mathrm{SO}_{4}$ receiving solution. The kinetic parameters for the successful transport were calculated and listed in Table 3.

\section{Conclusions}

This paper reports the development of the first PIM with a binary carrier consisting of PC-88A and Versatic 10 and its use

Table 3 Kinetic parameters for $\mathrm{Sc}^{3+}$ transport. Experimental conditions as in Fig. 8

\begin{tabular}{llllll}
\hline$K\left(\mathrm{~h}^{-1}\right)$ & $V\left(\mathrm{~m}^{3}\right)$ & $A\left(\mathrm{~m}^{2}\right)$ & {$\left[\mathrm{Sc}^{3+}\right]_{0}^{\mathrm{f}}\left(\mathrm{mol} \mathrm{L}^{-1}\right)$} & $P\left(\mathrm{~m} \mathrm{~h}^{-1}\right)$ & $J_{0}\left(\mathrm{~mol} \mathrm{~m}^{-2} \mathrm{~s}^{-1}\right)$ \\
\hline 0.0664 & $5 \times 10^{-5}$ & $4.9 \times 10^{-4}$ & $1 \times 10^{-4}$ & $6.77 \times 10^{-3}$
\end{tabular}


for the selective separation and pre-concentration of $\mathrm{Sc}^{3+}$ from nitrate solutions containing other REM ions. The use of the binary carrier allowed easier stripping of $\mathrm{Sc}^{3+}$ from the PIM and thus enabled the construction of a successful membrane transport system for $\mathrm{Sc}^{3+}$. The transport efficiency of the PIM was found to depend on both the membrane and receiving solution compositions which were optimized as $4 \mathrm{wt} \% \mathrm{PC}-88 \mathrm{~A}+$ $36 \mathrm{wt} \%$ Versatic 10, $40 \mathrm{wt} \%$ DOP and $20 \mathrm{wt} \%$ CTA for the PIM and $1 \mathrm{M} \mathrm{H}_{2} \mathrm{SO}_{4}$ for the receiving solution. The results obtained in this study illustrate the potential of binary carrier PIMs for the separation and pre-concentration of target chemical species.

\section{Conflicts of interest}

There are no conflicts.

\section{Acknowledgements}

This research was supported by a Grant-in Aid for Scientific Research (No. JP16K06830) from the Ministry of Education, Science, Sports, and Culture of Japan, the Environment Research and Technology Development Fund (ERTDF, grant no. 3-1710) from the Ministry of the Environment Japan, and Open Partnership Joint Project of the Japanese Society for the Promotion of Science (Grant No. AJ179065 (29-9111-t13)).

\section{References}

1 L. Pei, L. Wang, W. Guo and N. Zhao, J. Membr. Sci., 2011, 378(1), 520-530.

2 Y. Deng, B. Peng, G. Xu, Q. Pan, Z. Yin, R. Ye, Y. Wang and L. Lu, Mater. Sci. Eng., A, 2015, 639, 500-513.

3 Z. Zhao, F. Kubota, N. Kamiy and M. Goto, Solvent Extr. Res. Dev., Jpn., 2016, 23(2), 137-143.

4 K. P. Parhi, H. K. Park, C. W. Nam and J. T. Park, J. Rare Earths, 2015, 33(2), 207-213.

5 R. A. Kumbasar and S. Kasap, Hydrometallurgy, 2009, 95(1), 121-126.

6 R. M. Izatt, J. Inclusion Phenom. Macrocyclic Chem., 1997, 29(3), 197-220.

7 S. D. Kolev, Liquid Membranes, in Encyclopedia of Analytical Science, ed. P. Worsfold, A.Townshend and C. Poole, Elsevier, Amsterdam, 2005, pp. 531-538.

8 L. D. Nghiem, P. Mornane, I. D. Potter, J. M. Perera, R. W. Cattrall and S. D. Kolev, J. Membr. Sci., 2006, 251, 7-41.

9 M. O'Rourke, R. W. Cattrall, S. D. Kolev and I. D. Potter, Solvent Extr. Res. Dev., Jpn., 2009, 16, 1-12.

10 M. I. G. S. Almeida, R. W. Cattrall and S. D. Kolev, J. Membr. Sci., 2012, 415-416, 9-23.
11 S. C. Lee, J. D. Lamb, M. H. Cho, C. H. Rhee and J. S. Kim, Sep. Sci. Technol., 2000, 35(5), 767-778.

12 P. S. Kusumocahy, K. Sumaru, T. Iwatsubo, T. Shinbo, T. Kanamori, H. Matsuyama and M. Teramoto, J. Membr. Sci., 2006, 280(1), 73-81.

13 X. Meng, C. Wang, P. Zhou, X. Xin and L. Wang, Front. Environ. Sci. Eng., 2017, 11(6), 9.

14 C. F. Croft, M. I. G. S. Almeida, R. W. Cattrall and S. D. Kolev, J. Membr. Sci., 2018, 545, 259-265.

15 B. Mahanty, P. K. Mohapatra, D. R. Raut, D. K. Das, P. G. Behere, M. Afzal and W. Verboom, Ind. Eng. Chem. Res., 2016, 55(7), 2202-2209.

16 B. Mahanty, P. K. Mohapatra, D. R. Raut, D. K. Das, P. G. Behere, M. Afzal and W. Verboom, J. Membr. Sci., 2016, 516, 194-201.

17 C. Sgarlata, G. Arena, E. Longo, D. Zhang, Y. Yang and R. A. Bartsch, J. Membr. Sci., 2008, 323(2), 444-451.

18 I. Iben Nasser, F. Ibn El Haj Amor, L. Donato, C. Algieri, A. Garofalo, E. Drioli, et al., Chem. Eng. J., 2016, 295, 207-217.

19 S. P. Best, S. D. Kolev, J. R. Gabriel and R. W. Cattrall, J. Membr. Sci., 2016, 497, 377-386.

20 Y. Y. N. Bonggotgetsakul, R. W. Cattrall and S. D. Kolev, J. Membr. Sci., 2016, 514, 274-281.

21 D. Wang, R. W. Cattrall, J. Li, M. I. G. Almeida, G. W. Stevens and S. D. Kolev, J. Membr. Sci., 2017, 542, 272-279.

22 G. Arslana, A. Yılmazb, A. Torc and M. Ersozb, Desalin. Water Treat., 2017, 75, 348-356.

23 A. F. Shaaban, M. M. Azab, A. A. Mahmoud, A. A. Khalil and A. M. Metwally, Desalin. Water Treat., 2017, 70, 190-200.

24 D. Wang, J. Hu, D. Liu, Q. Chen and J. Li, J. Membr. Sci., 2017, 524, 205-213.

25 W. Wang and C. Cheng, J. Chem. Technol. Biotechnol., 2011, 86, 1237-1246.

26 M. Sharaf, W. Yoshida, F. Kubota and M. Goto, Proc. Int. Solvent Extr. Conf., 2017, 49-54.

27 Y. Baba, F. Kubota, M. Goto, R. W. Cattrall and S. D. Kolev, J. Chem. Technol. Biotechnol., 2016, 91(5), 1320-1326.

28 F. Kubota, Y. Shimobori, Y. Koyanagi, K. Shimojo, N. Kamiya and M. Goto, Anal. Sci., 2010, 26, 289-290.

29 E. Miyako, T. Maruyama, N. Kamiya and M. Goto, J. Am. Chem. Soc., 2004, 126, 8622-8623.

30 Y. Liu and M. S. Lee, Solvent Extr. Ion Exch., 2016, 34, 74-85. 31 N. Pereira, A. M. St John, R. W. Cattrall, J. M. Perera and S. D. Kolev, Desalination, 2009, 333, 236-327.

32 Y. Yıldız, A. Manzak, B. Aydin and O. Tutkun, Mater. Teh., 2014, 48(5), 791-796.

33 P. Ramakul, U. Mooncluen, Y. Yanachawakul and N. Leepipatpiboon, Ind. Eng. Chem., 2012, 18(5), 1606-1611. 